\title{
Les enjeux cognitifs du défi environnemental dans les exploitations agricoles
}

The Cognitive Issue of Environmental Challenge in Farms

\section{Audrey Rivaud et Jacques Mathé}

\section{(2) OpenEdition}

\section{Journals}

Édition électronique

URL : http://journals.openedition.org/economierurale/3026

DOI : 10.4000/economierurale.3026

ISSN : 2105-2581

Éditeur

Société Française d'Économie Rurale (SFER)

Édition imprimée

Date de publication : 23 mai 2011

Pagination : 21-35

ISSN : 0013-0559

\section{Référence électronique}

Audrey Rivaud et Jacques Mathé, « Les enjeux cognitifs du défi environnemental dans les

exploitations agricoles », Économie rurale [En ligne], 323 | mai-juin 2011, mis en ligne le 23 mai 2013, consulté le 30 avril 2019. URL : http://journals.openedition.org/economierurale/3026 ; DOI : 10.4000/ economierurale.3026 


\title{
Les enjeux cognitifs du défi environnemental dans les exploitations agricoles
}

\author{
Audrey RIVAUD • Centre de recherche sur l'intégration économique et financière (CRIEF), \\ Université de Poitiers et Cemagref Bordeaux \\ audrey.rivaud@univ-poitiers.fr
}

Jacques MATHÉ • Réseau CER-France et Centre de recherche sur l'intégration économique et financière (CRIEF), Université de Poitiers

jmathe@79.cerfrance.fr

\section{Introduction}

M algré la diversité des structures productives et des filières qui le constituent, le secteur agricole dans son ensemble est confronté, avec une importance grandissante depuis les années 1990, à la nécessité d'intégrer les enjeux environnementaux dans son mode de production. Toutefois, en France, près de vingt ans après les premières mesures agro-environnementales, les progrès en matière de préservation du milieu restent timides (Dupraz et Pech, 2007). Des travaux mettent en évidence un certain nombre de limites liées aux politiques publiques pour expliquer les difficultés des agriculteurs à prendre en compte les enjeux environnementaux dans les systèmes productifs, en évoquant, entre autres, la faiblesse relative de l'enveloppe financière consacrée aux politiques environnementales (Mollard et al., 2003 ; Bureau, 2007), ou en soulignant encore le caractère dual des orientations des politiques agricoles, avec d'un côté, la libéralisation du secteur et de l'autre, l'accroissement des normes, notamment environnementales (Allaire, 2002 ; Rivaud, 2008). En nous plaçant en complémentarité par rapport à ces analyses, nous souhaitons toutefois mettre en lumière des enjeux, moins souvent traités dans la littérature abordant la relation « agriculture-environnement », relatifs aux problèmes cognitifs que peuvent engendrer, pour les acteurs, les évolutions institutionnelles

En incitant ou en contraignant les pratiques au respect de normes environnementales, la rupture paradigmatique que connaît le secteur agricole depuis les années 1990-2000 a des implications directes au niveau des itinéraires techniques et nécessite une adaptation en termes de compétences. Elle conduit donc à poser la question du changement technique et organisationnel, et plus particulièrement à interroger les capacités des agriculteurs à s'approprier des innovations environnementales. À cet égard, nous avons à nous pencher sur l'organisation et la dynamique du système de production et de transfert de connaissances et de savoirfaire en agriculture, afin de comprendre dans quelles mesures les entreprises agricoles peuvent répondre aux exigences environnementales compte tenu de leur trajectoire cognitive.

Quelques travaux appliqués à des initiatives environnementales françaises (Dupeuble, 2005 ; Ansaloni, Fouilleux 2006 ; Del'Homme et al., 2008) attirent l'attention sur le rôle des déterminants cognitifs pour la mise en œuvre de techniques productives innovantes. Néanmoins, ces travaux se sont concentrés sur les modalités du changement technique dans le cadre de démarches environnementales volontaires. Nous souhaitons élargir le propos en nous intéressant à l'ensemble des politiques agro-environnementales, y compris aux enjeux liés l'application du volet réglementaire.

À la lumière des approches cognitivistes, qui placent la question des compétences au cœur de leur analyse du développement et de l'évolution des firmes, nous proposons dans la première partie de cet article, une lecture 
de la trajectoire cognitive des exploitations agricoles, en mettant en évidence de quelle manière celle-ci est étroitement liée au contexte institutionnel du secteur. En nous appuyant notamment sur les résultats d'une enquête de terrain réalisée auprès des agriculteurs de la région Poitou-Charentes ${ }^{1}$, cette première partie nous conduit à souligner un certain nombre de facteurs d'inertie liés aux caractéristiques des savoir-faire des agriculteurs, ces derniers subissant des effets de dépendance au sentier technologique. Après avoir mis en évidence les enjeux en termes d'acquisition de compétences nouvelles, nécessaires à l'amorce du processus de changement technique dans les exploitations, nous nous interrogeons dans la seconde partie, sur la dynamique de production et de diffusion d'innovation environnementales, en envisageant la dimension collective comme une voie à explorer. Sur la base de l'expérience québécoise des clubs-conseils en agro-environnement, nous proposons, à la suite, une réflexion sur les marges de manœuvre des politiques publiques françaises pour accompagner les agriculteurs confrontés au défi environnemental.

\section{Une lecture cognitiviste Compétences tacites et changement technique}

En considérant la firme comme un répertoire de routines et de compétences, les approches cognitivistes - théories des compétences et évolutionnistes -, se sont attachées à mettre en évidence le rôle essentiel des mécanismes cognitifs pour comprendre les trajectoires productives des firmes et les fondements du changement technique. À ce titre, les éléments analytiques qu'elles proposent nous permettent d'appréhender les formes

1. Les enquêtes par entretiens semi-directifs ont été plus précisément réalisées dans les départements de la Charente et de la Charente-Maritime, au printemps 2009 , auprès de 62 agriculteurs représentatifs de la population agricole régionale. des mutations dans l'agriculture au niveau des exploitations et la faculté de ces dernières à répondre d'un point de vue cognitif aux nouvelles exigences environnementales.

\section{Le rôle des compétences dans le processus du changement technique}

Les travaux relevant des approches cognitivistes ont pour point commun de rendre compte du pouvoir explicatif de la notion de compétence pour analyser l'organisation et le développement de la firme. Au cœur de ces approches, l'entreprise est caractérisée par une collection de « ressources » productives tangibles et intangibles (Penrose, 1959), ou de « compétences », ces compétences étant définies comme la somme des connaissances, expériences et savoir-faire propres à la firme et qui proviennent de ses activités passées (Richardson, 1972). Dès lors, la nature, le développement et la combinaison des différentes ressources internes sont à la base des performances des entreprises, mais également de leurs possibilités d'évolution.

Parmi ces approches, les travaux évolutionnistes (Nelson, Winter, 1982; Kline, Rosenberg, 1986 ; Dosi, 1988 ; Teece, Pisano, 1998), davantage centrés sur les problématiques du changement technique, soulignent la nécessité de prendre conjointement en considération les deux éléments que sont la Recherche \& Développement et l'apprentissage, et leur interaction. Ils ont donc cherché à compléter l'analyse des capacités d'évolution des firmes, en précisant la spécificité des compétences et en décryptant les modalités de leur accroissement. À la suite de Michel Polanyi (1966) qui soulignait l'écart entre la connaissance dont disposent les individus et la capacité à formuler les savoirs ${ }^{2}$, l'approche évolutionniste insiste sur le fait qu'une large part des compétences est tacite (Nelson, Winter,

2. "We know more than we can tell" (Polanyi, 1966). 
op. cit). Dès lors, ces ressources cognitives particulières ne peuvent pas être codifiées pour être diffusées librement et réutilisées de manière performante en dehors de l'organisation qui les détient, et elles sont mémorisées dans les routines de l'entreprise. Les routines, qui peuvent se définir comme un modèle répétitif d'action «a repetitive pattern of activity $»^{3}$, sont donc l'expression des compétences de la firme. Elles représentent sa mémoire organisationnelle (ibidem $)^{4}$. La composante tacite des compétences implique par conséquent que, si les actes répétés peuvent faire l'objet d'une amélioration et sont transmis par le partage d'expérience (Dosi, 1988), à l'inverse, les actes non répétés sont progressivement oubliés. MacKenzie et Spinardi (1995) ont mis en évidence ce processus de dépossession des compétences tacites non mobilisées, en s'appuyant sur l'exemple de la production d'armes nucléaires. Les auteurs expliquent qu'il suffirait d'une rupture d'une génération pour qu'un grand nombre de procédures non codifiables se délitent, conduisant ainsi à une «désinvention » de la bombe atomique.

Sur la base de ces concepts, les auteurs évolutionnistes révèlent le rôle central des différents mécanismes d'apprentissage comme moyen fondamental d'accroissement des ressources cognitives des firmes (Dosi, op. cit. ; Dosi et al., 1990 ; Teece et Pisano, op. cit.). L'apprentissage est envisagé comme le processus par lequel la répétition et l'expérimentation font que les tâches sont effectuées avec davantage d'aisance et de rapidité, et permet alors l'identification de nouvelles opportunités de production (Dosi et al., op. cit.). Il est perçu dans sa dimension interne à l'entreprise. L'apprentissage est donc l'élément moteur de la dynamique de l'innovation dans la firme évolutionniste, dans la mesure où en plus

3. In Nelson et Winter, 1982, p. 97.

4. Ibidem, p. 98. d'être mémorisées, les compétences utilisées régulièrement s'améliorent. Dans le même temps, en s'appuyant sur les ressources existantes, les mécanismes d'apprentissage conduisent la firme à se spécialiser dans des champs de compétences précis. $\mathrm{Ce}$ phénomène de spécialisation cognitive met en exergue l'inertie des routines : l'entreprise évolue le long d'une trajectoire technologique spécifique largement prédéfinie (Nelson et Winter, 1982 ; Dosi, op. cit.) qui limite ses capacités à mobiliser des domaines cognitifs trop différents. Dit autrement, la structure présente et passée de la firme influence ses capacités d'évolution et donc son processus d'innovation, et révèle la dynamique interne de l'entreprise de même que sa dépendance face à l'histoire. Les auteurs évolutionnistes soulignent alors l'existence d'une contrainte de sentier (path dependency) et reconnaissent le caractère ambivalent des compétences tacites : la spécialisation cognitive permise par l'accumulation des connaissances est à la base des performances de l'entreprise et de son avantage concurrentiel, mais elle enferme la firme dans une trajectoire technologique qui restreint ses possibilités de repositionnement cognitif.

Pour affiner encore la compréhension du changement technique, certains auteurs évolutionnistes insistent sur le rôle de l'environnement social et institutionnel dans les processus de prise de décision des entreprises, et donc dans le choix des compétences privilégiées. De ce fait, la capacité d'innovation ne dépend pas seulement des caractéristiques internes à la firme, mais aussi des différents espaces, institutionnel, relationnel et géographique, dans lesquels elle s'inscrit. Sans remettre en cause l'importance du learning by doing (Arrow, 1962), les processus de «learning by interacting » sont ainsi révélés, et avec eux la nécessité d'analyser le contexte socio-institutionnel (Lundvall, 2005) et de prendre en compte le caractère interactif du processus de changement technique (Porter, 1999). 
Les relations et les collaborations que la firme entretient, localement ou non, orientent sa trajectoire cognitive (Lundvall, op. cit.), et ses capacités d'apprentissage (knowledge capacities). De même, le contexte institutionnel des entreprises conditionne la dynamique de l'innovation.

Les éléments des théories cognitivistes permettent donc d'appréhender la dynamique du changement technique en mettant en évidence le rôle des compétences et de l'apprentissage dans la détermination de la trajectoire technologique des firmes. Ils révèlent, notamment, l'existence de processus de spécialisation cognitive et de sentier de dépendance. Ils permettent, en outre, d'intégrer des déterminants relatifs au contexte institutionnel et social dans lequel évoluent les entreprises pour comprendre la dynamique de l'innovation. Ces éléments nous semblent apporter des clés de compréhension aux problématiques qui se posent actuellement dans les exploitations agricoles confrontées à la prise en compte des enjeux environnementaux dans leurs systèmes productifs. Les outils évolutionnistes, plus particulièrement, nous invitent à nous intéresser à l'évolution des compétences dans les exploitations d'une part, et à prendre en considération les dimensions institutionnelles qui sous-tendent la dynamique cognitive dans le secteur agricole, d'autre part.

\section{La dynamique cognitive des exploitations agricoles françaises}

Afin de comprendre dans quelles mesures les entreprises agricoles peuvent mettre en place des pratiques favorables à l'environnement, nous cherchons au préalable à rendre compte de la dynamique cognitive qui s'est mise en œuvre dans le secteur agricole français. Nous proposons pour cela d'adopter une lecture à un double niveau, en mettant en évidence les évolutions en termes de savoirfaire à l'échelle de l'exploitation agricole, et en indiquant, à l'échelle sectorielle, de quelles manières ces évolutions sont le fruit à la fois de compromis institutionnalisés établis entre les agriculteurs et la société et de la structuration de la profession agricole. Ce positionnement nous amène à présenter les éléments relatifs aux processus de production et de transfert des innovations en agriculture qui, en dépit de l'hétérogénéité des systèmes productifs, ont conduit à une convergence relativement forte de la dynamique des ressources cognitives, dans le but de répondre à un objectif commun d'accroissement des rendements.

Traditionnellement, l'activité agricole requiert pour sa réalisation des compétences en termes d'adaptation et d'adaptabilité, en raison du caractère vivant des productions adaptation aux conditions climatiques, agronomiques ou zootechniques. Cette spécificité implique qu'une large part des savoirfaire des agriculteurs était construite sur l'observation de la culture ou du troupeau, et dépendait donc du contexte de chaque exploitation. Basée sur des compétences essentiellement tacites (Allaire, Wolf, 1999), la production de connaissances nouvelles en agriculture se réalisait à travers la mise en commun d'expériences, lors de la constitution de groupes professionnels locaux dont l'objet était la réalisation collective de certaines pratiques (Darré, 1996) ${ }^{5}$ La somme des savoir-faire implicites pouvait, dès lors, faire l'objet d'une décontextualisation permettant la codification et donc le processus d'explicitation des compétences - ces dernières revêtant alors un caractère transférable. Pour définir ce processus, Nonaka et al. (2000) utilisent le concept d'externalisation des compétences tacites. Dans le secteur agricole, si l'externalisation était amorcée dans le cadre des communautés de pratiques, elle se réalisait toutefois plus complètement au sein des réseaux de groupes professionnels, tels les Coopératives d'utilisation de matériel agricole

5. On fait notamment référence à la constitution de chantiers d'entraide pour réaliser des tâches telles que l'ensilage ou encore la fenaison. 
(CUMA) ou les Centres d'études techniques agricoles CETA (CETA) pour ne donner que quelques exemples (Dupeuble, op. cit.). Dans ce schéma, les connaissances tacites à la base de l'amélioration des itinéraires techniques étaient produites par les agriculteurs eux-mêmes, alors que les connaissances codifiées étaient formalisées par les techniciens agricoles (Labarthe, 2005). S'appuyant sur ces connaissances formalisées, la mise en place d'un travail de recherche pouvait donner lieu à des innovations et donc à de nouveaux savoirs explicites. Ces derniers faisaient l'objet d'une transmission dans les exploitations à travers les conseils fournis par les techniciens. Ainsi, le processus de production et de transfert de connaissances se réalisait à l'appui d'un double mécanisme de formalisation de savoir-faire tacites d'une part et d'internalisation de connaissances explicites d'autre part.

Néanmoins, cette dynamique est progressivement bouleversée à partir des années 1960, avec l'émergence d'une nouvelle configuration institutionnelle. Les politiques agricoles qui voient le jour - notamment la politique agricole commune en 1962 - se fondent sur un compromis établi entre les agriculteurs et l'État, comportant un objectif commun à l'ensemble des entreprises agricoles : optimiser les rendements afin d'accroître la production et de garantir la sécurité alimentaire. Dans le même temps, la structuration de la profession autour des organisations professionnelles et syndicales, assure la diffusion des principes du modèle productiviste. Le régime de fonctionnement (Bartoli, Boulet, 1990) qui se met alors en place a engendré une modification des trajectoires cognitives des exploitations agricoles. Afin de garantir l'augmentation de la productivité, les agriculteurs se sont plus ou moins progressivement engagés sur la voie de la spécialisation, caractéristique du régime d'accumulation intensif fordiste des Trente Glorieuses (Allaire, 1988). Alors que la politique de « réforme des structures » a créé les conditions d'une sélection sévère des exploitations familiales, entrâ̂nant des dommages sociaux importants, les agriculteurs répondant aux exigences de la modernisation se sont transformés en ouvriers spécialisés (Jollivet, 2007). Les innovations, dynamisées par l'introduction des outils issus du complexe de l'agrofourniture - intrants, alimentation animale, mécanisation, etc. - , ont été orientées vers la production de références techniques standardisées, applicables au plus grand nombre d'exploitations. Le processus de production de connaissances s'est donc peu à peu affranchi du dialogue entre l'apprentissage fondé sur les compétences tacites et la production de compétences codifiées, pour privilégier les savoirs explicites. Ces derniers ont été transmis, en France, par l'intermédiaire des organismes de vulgarisation (Labarthe, op. cit.), et des organisations professionnelles au sens large du terme dont le rôle dans la diffusion des connaissances et la construction des compétences a lui aussi été structurant. L'accroissement de la formalisation s'est alors traduit par une augmentation de savoir-faire répétitifs. Il s'agissait pour les agriculteurs d'appliquer un ensemble de préconisations, de codes d'utilisation, qui ont conduit à un affranchissement des spécificités liées au vivant. En effet, la maîtrise d'un certain nombre d'éléments aléatoires - relatifs aux maladies des plantes notamment - a considérablement sécurisé les performances agricoles. $\mathrm{La}$ dynamique de type top down des ressources cognitives a alors permis l'acquisition de compétences particulièrement appropriées aux objectifs d'optimisation et d'intensification du modèle agricole.

Cette trajectoire cognitive socialement sélective, marquée par des investissements massifs pour la création de références techniques standardisées, s'est accompagnée d'un abandon progressif des compétences liées à l'observation et à l'adaptabilité au contexte, qui incarnaient la spécificité et la complexité des savoir-faire en agriculture. En effet, en étant de moins en moins mobi- 
lisées, ces compétences ont peu à peu disparu des routines des entreprises agricoles. Les agriculteurs ont donc été confrontés, en quelque sorte, à une dépossession de certains de leurs savoir-faire tacites. Or ces savoir-faire tacites oubliés représentent une des clés de voute de la mise en œuvre d'un ensemble de normes agro-environnementales qui s'imposent au monde agricole.

\section{Le sentier de dépendance et le défi des pratiques agro-environnementales}

À partir des années 1990, un certain nombre de facteurs de déstabilisation, internes et externes $^{6}$, implique une modification des rapports institués entre les agriculteurs et la société. Bien qu'il soit encore difficile de définir aussi précisément que pour la période précédente les termes d'un compromis institutionnalisé, les bases d'un nouveau paradigme agricole se dessinent autour de deux lignes directrices :

- d'un côté, le retour à une régulation concurrentielle, passant par la diminution d'un certain nombre d'entraves aux échanges internationaux,

- de l'autre, la prise en compte du rôle à jouer par l'agriculture en termes de maintien du dynamisme rural, d'entretien et de préservation de l'environnement.

L'émergence de ce paradigme conduit à poser d'une manière différente la question de la performance agricole, préalablement définie sur la base de critères techniques essentiellement et économiques, en y incluant notamment la dimension environnementale (Rivaud, 2008). Dès lors, la

6. Ces facteurs concernent l'inadéquation entre la production et la demande, les coûts de stockage liés aux surproductions, la croissance des dépenses budgétaires en dépit d'une incapacité à maintenir le revenu des agriculteurs, les remises en cause de la politique communautaire par les partenaires commerciaux de l'UE, les crises sanitaires à répétition et les dégradations de l'environnement engendrées par l'intensification des techniques de production. modification des objectifs assignés aux agriculteurs se traduit par la nécessité de mobiliser des savoir-faire complexes pour répondre à des enjeux multidimensionnels. Les pratiques jusqu'alors considérées comme les indicateurs d'excellence de l'agriculture moderne sont incriminées (Jollivet, 2007). La question du changement technique et organisationnel se pose d'autant plus que les politiques agro-environnementales influencent directement les itinéraires techniques des exploitations agricoles, que ce soit dans le cadre des démarches environnementales volontaires ou dans le cadre des mesures obligatoires. Par exemple, en réduisant les possibilités d'utilisation des intrants, les pratiques de traitements systématiques et préventifs sont remises en cause.

Cependant, au regard de la trajectoire technologique empruntée par les exploitations agricoles au cours de la période 19601990, il semble que le repositionnement cognitif attendu pour intégrer les enjeux environnementaux soit freiné par l'existence d'un certain nombre d'inerties, à la fois internes aux exploitations et liées à l'environnement des agriculteurs. Sans ignorer les contraintes financières engendrées par l'adoption d'innovations à caractère environnemental - les coûts privés impliquant un bénéfice public (Gasmi, Grolleau, 2003) -, ou encore des problèmes de compatibilité au sein des orientations de la gouvernance sectorielle - entre libéralisation et montée en puissance de la normalisation -, nous soutenons, pour notre part, l'idée que les agriculteurs sont confrontés à des limites en termes de compétences, pour la mise en œuvre des normes environnementales dans les systèmes productifs.

En reprenant les termes de l'analyse évolutionniste, la spécialisation cognitive des entreprises agricoles autour d'une production unique et d'un ensemble de savoir-faire répondant aux exigences du modèle d'optimisation des systèmes productifs, a conduit les agriculteurs sur un sentier de dépendance 
qui contraint aujourd'hui leur positionnement stratégique. L'inertie des routines et la dépossession de certaines compétences tacites limiteraient la capacité des exploitants à s'approprier les connaissances produites par la recherche agronomique en matière d'itinéraires techniques à dominante environnementale. En effet, pour reprendre l'exemple de la restriction des produits de traitement utilisés dans les cultures, si des procédés sont élaborés dans des organismes tels que l'INRA ou les instituts techniques, ils nécessitent pour leur mise en œuvre que les agriculteurs se réapproprient des compétences liées à l'observation dans les parcelles et au suivi de cultures in situ. Confrontés à un déficit de savoir-faire, les producteurs agricoles, notamment ceux installés dans les années 1980-1990, associent ce type de normes environnementales à un accroissement du risque pour la conduite de leur campagne (Dron et al., 2003). Les quelques éléments d'investigations empiriques dont nous disposons illustrent l'hypothèse que nous défendons.

Dans le cadre des enquêtes réalisées auprès d'agriculteurs de la région PoitouCharentes $^{7}$, le premier frein cité par les professionnels concernant la prise en compte des enjeux environnementaux dans les systèmes productifs se référait, effectivement, au risque engendré en termes d'instabilité des rendements de la production. $\mathrm{La}$ prépondérance de la question des rendements documente le fait que les agriculteurs se trouvent dans une impasse technique relative, dans la mesure où ils envisagent difficilement des itinéraires productifs fondés sur des références différentes de celles qu'ils connaissent déjà. Par ailleurs, près d'un tiers des agriculteurs rencontrés ont évoqué plus directement les freins cognitifs, en expliquant que les normes environnementales nécessitaient l'appropriation de savoirs spécifiques différents des savoir-faire dont ils disposaient.

7. Voir Rivaud, 2010
Finalement, les contraintes environnementales posent des limites à la compétitivité des exploitations, lorsque celle-ci est appréhendée à travers le prisme de l'augmentation des rendements physiques (Ansaloni, 2009).

Au-delà de l'inertie des routines organisationnelles internes aux entreprises agricoles, les effets de sentier se renforcent également par l'existence de freins sectoriels au changement technique : les structures de formation (Mollard et al., op. cit.), les organismes de conseil agricole (Labarthe, op. cit.), mais aussi au sein des filières, les industries amont et aval, se référent encore au modèle intensif. En outre, la difficulté des mesures agro-environnementales (MAE) à trouver une audience en France s'explique par le pouvoir de résistance dont fait preuve le syndicalisme majoritaire, qui appréhende les MAE comme une négation du statut professionnel de producteur agricole relégué à des fonctions d'entretien des paysages (Alphandéry, Deverre, 1994). Dans ce cadre, émergent des blocages cognitifs liés aux « images », pour reprendre la terminologie de Penrose (1959), que les agriculteurs ont de leurs opportunités de développement, ces images relevant à la fois de constructions dépendantes de l'expérience, mais aussi de la façon dont les acteurs appréhendent l'environnement dans lequel ils évoluent. Ainsi, le contexte social incarne un frein supplémentaire au changement technique (Ansaloni, Fouilleux, 2006).

La mobilisation de la grille de lecture évolutionniste permet de souligner le fait que le modèle agricole issu de la période modernisatrice a entraîné les exploitants sur un sentier de dépendance, qui limite aujourd'hui leur capacité interne d'apprentissage pour répondre aux attentes de repositionnement technique. Si les normes produites dans le cadre des politiques agroenvironnementales représentent des ressources cognitives codifiées - à l'instar des cahiers des charges - à la disposition des agriculteurs (Dupeuble 2005), l'application de ces normes met en lumière la 
problématique des modalités d'appropriation de compétences nouvelles, et de l'affranchissement des schémas automatisés propre au modèle productiviste. En d'autres termes, l'identification des freins cognitifs d'ordre microéconomiques pose avec vigueur la question des moyens externes à l'exploitation agricole de dépassement des freins internes et interroge, de ce fait, les formes de soutien à l'innovation à mettre en œuvre, afin d'atteindre les objectifs du nouveau paradigme agricole.

\section{Quelles modalités d'appropriation de compétences nouvelles pour l'adoption des normes environnementales ?}

S'intéresser à l'innovation en agriculture implique de prendre en considération la complexité du processus et la diversité des acteurs impliqués, comme le proposent Vanloqueren et Baret (2009), à travers une analyse en termes de Systèmes d'Innovation $^{8}$. En focalisant ici notre attention sur une sous-partie de ce système, relative à l'appropriation de compétences nouvelles à l'échelle de l'exploitation agricole, nous reprenons à notre compte les conclusions des auteurs concernant l'importance de l'intervention publique pour dépasser les situations de lock-in technologique, ces dernières limitant les capacités d'intégration des enjeux environnementaux. Avant d'identifier des pistes de réflexion concernant les actions à mettre en œuvre pour favoriser l'intégration les enjeux environnementaux, il convient de préciser les mécanismes qui favorisent l'appropriation par les agriculteurs de nouvelles ressources cognitives. Dans cette perspective, nous envisageons la dimension collective des processus d'apprentissage comme une des modalités de réponse au phénomène de dépendance de

8. L'analyse des SI fait référence aux travaux des évolutionnistes tenants d'une approche institutionnaliste (Lundvall, 1992, notamment). sentier préalablement révélé. Nous nous interrogeons, alors, sur les marges de manœuvre des politiques publiques pour accompagner les agriculteurs à travers le soutien au développement de la vie collective. Pour illustrer nos propos, nous rendons compte de l'expérience québécoise des clubs conseils en agro-environnement.

\section{Le retour sur la nature interactive et collective du processus d'appropriation de compétences}

En soumettant des prescriptions de pratiques contraignantes formalisées, les normes environnementales préfigurent un apprentissage futur. Toutefois, compte tenu de leur niveau de généralité, elles nécessitent de réaliser un travail d'adaptation au contexte spécifique de chaque exploitation agricole. La diversité des processus biologiques, par exemple, implique que les effets sur le milieu naturel de l'application d'une norme soient fortement variables dans le temps et dans l'espace. Afin d'atteindre les objectifs fixés par ces normes en termes de préservation de l'environnement, les agriculteurs doivent développer des compétences spécifiques en lien avec les caractéristiques de leur outil de travail. Ce mécanisme renvoie au questionnement central de notre analyse en interrogeant le processus de conversion de connaissances codifiées en compétences tacites (Dupeuble, 2005). En reprenant le principe de conversion des savoirs proposé par Nonaka et al. (2000), si la contextualisation est un pré-requis à l'appropriation de savoir-faire implicites, la création de compétences nouvelles qu'elle engendre permet en retour, dans le cadre d'une socialisation, d'engager une dynamique d'externalisation de ces savoir-faire, ayant pour effet d'améliorer ou de préciser les ressources cognitives codifiées - mouvement de décontextualisation.

Cependant, le double phénomène de contextualisation et de décontextualisation constitutif du processus d'apprentissage, se réalise à travers des changements d'échelle 
successifs, tant au niveau spatial que temporel, que seules les organisations collectives sont en mesure d'assurer (Dupeuble, 2005). Dit autrement, la dynamique de conversion des savoirs implique une dimension collective et justifie l'intérêt que nous y portons, dans la mesure où elle révèle le rôle des organisations de producteurs, souvent dotées de techniciens-conseils, pour faciliter le développement des interactions essentielles à l'appropriation des compétences (Lundvall, 2005).

Un certain nombre de travaux traitant de la problématique du changement technique en agriculture dans le cadre de la mise en œuvre volontaire de démarches environnementales, démontrent effectivement l'importance du collectif dans le processus de création de ressources nouvelles. Que ce soit à travers, l'examen des CTE/CAD Contrats Territoriaux d'Exploitation / Contrats d'Agriculture Durable - (Dupeuble, 2005) ou l'analyse de l'hybridation technique des exploitations laitières bretonnes (Ansaloni, Fouilleux, 2006), ou encore l'étude d'un procédé innovant pour la protection du vignoble ${ }^{9}$ dans les exploitations viticoles bordelaises (Del'Homme et al., 2008), l'existence de groupes d'échanges, formels ou informels, est appréhendé comme une solution potentielle pour lever certains freins associés aux innovations environnementales et favoriser les processus d'apprentissage. Ces groupes d'échanges représentent donc l'un des vecteurs de la mise en application de pratiques favorables à l'environnement :

- d'une part, ils facilitent le transfert de référentiel technique,

- d'autre part, ils favorisent les efforts de conception intellectuelle pour la recherche des pratiques les plus performantes, à travers la mutualisation des savoirs et des savoir-faire.

9. Il s'agit de l'élaboration d'une nouvelle règle de décision conçue par l'équipe des pathologistes de l'INRA : Mildium ${ }^{\circledR}$.
La dimension collective, qui apparaît comme support de l'appropriation de compétences nouvelles, pourrait être une modalité de soutien pour l'application des politiques agro-environnementale relatives au volet facultatif - de type MAE - et au volet obligatoire. Cependant, si des initiatives collectives professionnelles ou locales existent, elles se révèlent à l'heure actuelle limitées au regard de leur diffusion. De surcroit, la concentration des exploitations et l'autonomisation/individualisation des procédés de production qui découlent du modèle modernisateur, restreignent les possibilités d'interactions avec le voisinage. À ces difficultés s'ajoute le fait que l'application de la réglementation environnementale dans des systèmes productifs dont les références, en termes de performance, sont celles de l'optimisation de l'outil de production, peut se traduire par des pertes économiques conséquentes et potentiellement vecteur de remise en cause de la pérennité des systèmes en question. Au-delà des enjeux environnementaux sous-jacents à l'avènement d'un nouveau paradigme agricole, ce sont des enjeux sociaux attachés au maintien des exploitations qui émergent

En nous référant aux dispositifs innovants instaurés dans le cadre de la politique agro-environnementale au Québec, ces dispositifs ayant fait l'objet d'investigations sur le terrain à travers la réalisation d'entretiens à dire d'experts, nous soulignons toutefois l'existence de marges de manœuvre pour les politiques publiques, pour accompagner les exploitants agricoles dans les processus d'acquisition de compétences agro-environnementales.

\section{L'expérience québécoise des clubs- conseils en agro-environnement}

Les politiques agro-environnementales québécoises se sont saisies, aux prémisses de leur élaboration, de la question des compétences et de leur appropriation par les exploitants. En reliant, d'une part, la réglementation environnementale aux connaissances 
acquises sur le terrain par des agriculteurspionniers et en déployant, d'autre part, un certain nombre d'aides dédiées à la recherche, à l'innovation technologique et à la formation des producteurs et des agronomes, ces politiques ont avant tout cherché à accompagner, d'un point de vue agronomique, les agriculteurs dans la modification de leur système productif en faveur de l'environnement. Historiquement, les premières initiatives agro-environnementales voient le jour au Québec au début des années 1980 avec la création de modèles précurseurs de services-conseils en agroenvironnement qui assuraient la formation des producteurs dans plusieurs régions du Québec. Ces expériences ont donné lieu en 1993 à la création des clubs-conseils en agro-environnement (CCAE), dans le cadre de la mise en place du Plan Vert.

Le fonctionnement des CCAE s'apparente à celui d'un CETA en France. Chaque structure est autonome et regroupe sous une forme associative 20 à 30 exploitations. Toutefois, à la différence des CETA, elle bénéficie d'un soutien public. Le club embauche un ingénieur-agronome, chargé d'accompagner les agriculteurs en vulgarisant des pratiques respectueuses de l'environnement. L'agronome a donc un rôle de producteur de références et savoir-faire, soit à travers sa propre expérimentation, soit en transférant et/ou en adaptant les travaux d'organismes de recherches, soit en coproduisant avec les agriculteurs les compétences et techniques répondant à la réglementation environnementale. L'aide financière apportée par les pouvoirs publics couvre la formation, la rémunération et les frais inhérents aux services de l'ingénieurconseil. En contrepartie de ce soutien, le CCAE se doit de remplir trois missions fondamentales.

- La première mission a trait à la réalisation d'une veille technologique et correspond donc à une production de références et de connaissances en amont. Elle s'appuie sur les liens qui existent entre les instituts de recherche, majoritairement universitaires, et les expérimentations sur le terrain réalisées par les agronomes-praticiens. En établissant un premier niveau de codification et de références techniques standardisées capacité des sols à recevoir les effluents d'élevage par exemple -, cette mission impulse l'évolution des pratiques agricoles. - La deuxième mission renvoie au rôle pédagogique des CCAE. Elle est largement facilitée par la structure de fonctionnement des groupes, qui allie la production individuelle de connaissances empiriques par les exploitants, à la confrontation collective et à l'animation-synthèse de l'agronome-conseil. Dans ce cadre, le groupe apparaît une fois encore comme le support de la vulgarisation et du transfert de compétences à l'ensemble des producteurs-membres.

- La troisième mission des clubs-conseils se rapporte au rôle d'évaluation. Les actions menées dans les exploitations font l'objet d'un bilan. Ce dernier permet de préciser la liste des actions à réaliser au cours des prochaines années et les orientations que le producteur doit prendre à moyen terme en matière d'agro-environnement.

En dix ans, les pratiques agricoles ont évolué de façon significative en faveur de la préservation de l'environnement: le surplus de phosphate dans les exploitations a été diminué de $78 \%$; depuis 2004, l'épandage des engrais phosphatés a été réduit de $25 \%$; de 2001 à 2006, la quantité d'herbicide utilisée a baissé de plus de $30 \%$ et les surfaces cultivées sans herbicide représentent $20 \%$ des hectares de cultures ; en ce qui concerne la conservation des sols, plus de $40 \%$ des surfaces cultivées sont exploitées sur la base de techniques simplifiées, c'està-dire, sans labour (Clubs-conseils en agroenvironnement, 2009). L'expérience québécoise est une illustration des modalités d'accompagnement des producteurs dans la modification de leurs pratiques productives. Les pouvoirs publics ayant identifié le rôle des compétences, ils ont fourni un soutien financier pour le développement 
des CCAE. Le succès de ces expériences, tant au niveau économique qu'environnemental, nous amènent à questionner les dispositifs français de soutien pour la mise application des politiques agro-environnementales pour assurer la diffusion d'un nouveau modèle productif.

\section{Les enjeux en termes de politiques publiques en France}

La situation française en termes d'enjeux relatifs à la préservation de l'environnement est très proche de la situation québécoise. Toutefois, la structuration des politiques publiques, plus particulièrement des politiques de soutien, diffère notablement. Il nous semble important de réaliser un retour sur l'organisation et le fonctionnement des orientations publiques françaises en matière d'environnement, afin d'identifier plus précisément les éléments institutionnels de blocage, qui ne permettent pas de pallier les problèmes cognitifs internes aux exploitations agricoles, et qui peuvent expliquer les performances mitigées des exploitations françaises sur le plan environnemental. Ces orientations s'articulent autour de deux volets que nous avons eus l'occasion d'évoquer : un volet facultatif, recouvrant un ensemble de démarches dans lesquelles les agriculteurs ont le choix de s'engager ou non pour modifier leurs pratiques, moyennant une rétribution financière - nous avons mentionné plus haut certaines de ces démarches et les conditions de leur réussite (II.1.) - ; et un volet obligatoire auquel chaque agriculteur doit se soumettre, qui fait l'objet d'une accentuation avec la mise en place des recommandations du Grenelle de l'environnement et du plan «écophyto ». Nous portons plus particulièrement notre attention sur ce deuxième volet en nous focalisant sur les moyens consacrés à sa mise en œuvre.

Les agriculteurs français sont soumis à une double réglementation : la réglementation issue de l'Union européenne qui s'est renforcée au cours des années 2000 avec l'instauration de la conditionnalité des $\operatorname{aides}^{10}$, à laquelle s'ajoutent les règlements mis en place par l'État. Ces dispositifs de régulation des pratiques agricoles ont en premier lieu des conséquences financières directes, la mise aux normes nécessitant des investissements matériels en partie supportés par les agriculteurs. Ils ont en second lieu, nous avons insisté à ce propos, des incidences sur la maîtrise des itinéraires productifs - savoir-faire - impliquant de manière plus indirecte des pertes économiques potentielles.

Actuellement, l'essentiel des ressources financières publiques à destination des agriculteurs est orienté vers un soutien aux investissements matériels - notamment à la rénovation des bâtiments. Les aides de l'État concernant les investissements immatériels liés à l'acquisition de compétences (formation, ingénierie, investissement dans des programmes de recherche sur des pratiques alternatives) sont très développées. S'il existe certaines opérations d'accompagnement $\left(\right.$ CIVAM $^{11}$ dans l'Ouest par exemple), cellesci restent limitées et elles peinent à se diffuser largement. Bien que la recherche et l'expérimentation continuent de bénéficier des aides publiques, leur transposition sur le terrain est de plus en plus souvent assurée par des organismes privés (coopératives, conseil privé, etc.). De ce fait, quand le conseil agroenvironnemental représente un coût direct pour l'entreprise dont le bénéfice est collectif, l'incitation à investir dans l'acquisition de nouvelles compétences nécessaires à l'application de la réglementation environnementale est réduite, sauf dans le cas où les mesures sont très coercitives.

Outre les conditions de financement pour l'acquisition de ces compétences spécifiques, il convient également de poser la

10. La conditionnalité soumet le versement de certaines aides européennes au respect d'exigences en matière d'environnement, de santé et de bien être des animaux.

11. Centre d'initiatives pour valoriser l'agriculture en milieu rural. 
question des conditions de l'essaimage des nouveaux savoir-faire. La densité des exploitations, les modalités d'exercice du métier isolement des producteurs et astreinte de travail -, le désengagement des organismes parapublics et notamment des Chambres d'Agriculture, mais surtout le syndicalisme majoritaire qui, à travers une tradition de cogestion freine la mise en œuvre des processus de conversion des savoirs et leur diffusion à grande échelle, sont autant d'éléments qui ne permettent plus de reproduire les méthodes de vulgarisation qui ont fait le succès du modèle productiviste et d'engendrer les processus d'appropriation des compétences. De plus, les acteurs qui assurent aujourd'hui le conseil technique aux entreprises agricoles, issus du complexe de l'agrofourniture et des instituts techniques ont, à l'image des agriculteurs, une trajectoire cognitive fortement empreinte des références du modèle modernisateur. L'évolution de la structure du transfert de connaissances, entre privatisation et sentier de dépendance, renforce l'inertie liée aux propres freins cognitifs des agriculteurs, et limite d'autant plus les capacités d'adaptation aux politiques agro-environnementales.

Au regard de ces éléments, il apparaît que la réglementation remet en cause toute la chaîne de production et de transfert des compétences. Dans ce cadre, les gestionnaires de la politique agricole notamment ont à réfléchir sur des modalités originales à concevoir pour accompagner plus globalement les agriculteurs dans le processus de changement technique qui s'impose à eux, et sur les acteurs en mesure d'assurer ces fonctions d'accompagnement et de vulgarisation. Ayant insisté à plusieurs reprises sur la dimension collective du processus d'apprentissage, il est possible de concevoir des formes nouvelles de conseil collectif, en s'inspirant par exemple des organisations québécoises, basées sur un mode de fonctionnement privé, mais encouragées par l'existence d'incitations financières, sous réserve de procédures d'évaluations - dimi- nution des impacts environnementaux.

Certains acteurs déjà en place, à l'instar des coopératives, pourraient assumer, sur la base d'un encouragement financier, cette mission d'accompagnement. En effet, compte tenu de leur inscription territoriale et de leur rôle d'interface entre les producteurs et les circuits de commercialisation, les coopératives se trouvent fortement concernées par les enjeux environnementaux et elles offrent le cadre collectif qui permet de dynamiser la création de ressources cognitives (Filippi, Triboulet, 2006 ; Del'Homme et al., 2008). Néanmoins, d'autres formes d'organisations, comme les structures associatives, sont envisageables.

Si les formes des dispositifs d'accompagnement à mettre en place sont encore à modeler, autour des principes du collectif et de l'instauration de soutiens financiers comme le suggèrent nos précédents propos, il persiste toutefois des enjeux relatifs à la transformation des structures professionnelles et syndicales pour dépasser les situations de lock-in identifiées au niveau des exploitations agricoles et à l'échelle du secteur. En effet, fortement opposées à l'affaiblissement du modèle productiviste qui a fait leur succès, les organisations professionnelles renforcent l'inertie à la fois individuelle et collective face à l'avènement des problématiques environnementales.

Enfin, en amont de l'entreprise agricole, le défi environnemental se pose également aux ingénieurs agronomes et à leur capacité à produire le degré de connaissances nécessaire à la sécurisation des itinéraires techniques intégrant les problématiques environnementales (Vanloqueren, Baret, 2009). En effet, les parcours alternatifs à l'agriculture productiviste ayant longtemps été décriés par les différents acteurs du secteur et par les pouvoirs publics qui consacraient l'essentiel des ressources financières aux pratiques conventionnelles, les techniciens ou ingénieurs travaillant sur ces pratiques alternatives n'ont trouvé en France qu'une faible audience. 


\section{Conclusion}

Les politiques agro-environnementales, en soumettant des prescriptions de pratiques contraignantes, impliquent pour leur adoption une modification des savoir-faire dans les exploitations agricoles. Les questionnements relatifs à leur mise en œuvre supposent dès lors la production d'une réflexion sur les capacités cognitives des agriculteurs à opérer le changement technique attendu. En nous appuyant sur les développements théoriques relevant des approches cognitivistes, nous avons cherché dans cet article à mettre en évidence l'existence de blocages au sein des entreprises agricoles, quant à l'appropriation de techniques de production plus favorables à l'environnement. En effet, la spécialisation cognitive opérée au cours de la période modernisatrice, de même que le recours aux outils issus du complexe de l'agrofourniture, ont conduit les agriculteurs sur un sentier de dépendance qui lie la performance agricole à l'augmentation des rendements physiques. Dépossédés d'un certain nombre de compétences tacites relatives à l'observation et au suivi in situ des productions, les agriculteurs semblent se trouver dans une impasse cognitive face à l'avènement d'un nouveau paradigme agricole. Les outils théoriques que nous mobilisons nous conduisent donc à mettre en évidence l'existence de freins puissants à l'intérieur des exploitations au développement d'un modèle de production intégrateur des enjeux environnementaux. Prenant acte de la timidité des progrès réalisés en matière de préservation de l'environnement, nous défendons l'idée que les dimensions relatives aux compétences au sein des entreprises agricoles représentent une des clés de voute de la réussite des programmes environnementaux. Toutefois, les matériaux empiriques que nous avons mobilisés pour illustrer nos propos revêtent une dimension exploratoire et mériteraient de faire l'objet d'investigations plus systématiques.

Le cadrage théorique nous conduisant à révéler des phénomènes de lock-in au niveau des exploitations agricoles, nous nous sommes questionnés sur les modalités de dépassement de ces situations de blocage. Une des pistes que nous avons identifiées a trait à la mise en œuvre de démarches collectives pour amorcer le processus d'appropriation de compétences nouvelles. En nous référant aux expériences françaises relatives à l'engagement en faveur de démarches environnementales volontaires ou à l'exemple québécois des clubs conseil en agro-environnement, la dimension collective paraît incarner un moyen de sécurisation et d'accroissement des savoirs. Néanmoins, les difficultés relatives la mise en place de ces démarches collectives à grande échelle en France, semblent limiter l'amorce d'un processus de conversion des savoirs.

Finalement, l'intérêt de la démarche analytique développée dans ce travail sur la relation agriculture-environnement, réside certainement dans la clarification des enjeux en termes d'intervention publique relative au soutien à l'appropriation de compétences nouvelles. Néanmoins, ces enjeux sont à mettre en perspective des modalités de diffusion d'un nouveau modèle agricole par le biais des structures professionnelles. L'étude du poids de ces structures et de leur rôle dans l'orientation du modèle productif agricole apporterait des éclairages complémentaires pour approfondir la compréhension des facteurs d'inertie déjà identifiés. 


\section{RÉFÉRENCES BIBLIOGRAPHIQUES}

Allaire G. (1988). Le modèle de développement agricole des années 1960. Économie rurale, p. 171-181.

Allaire G. (2002). Les compétences professionnelles agricoles et les défis de la multifonctionnalité. In "Loi d'orientation agricole, savoirs et qualifications professionnelles, analyse des enjeux et perspectives pour l'évolution des savoirs », Dijon, Educagri, p. 151-163.

Allaire G., Wolf S.-A. (1999). Collective goods and accountability in technical information system. $17^{\text {th }}$ Congress of European Society of Rural Sociology, August.

Alphandéry P., Deverre C. (1994). La politique agri-environnementale communautaire et son application en France. INRA Sciences Sociales, $\mathrm{n}^{\circ}$ 2-3, p. 1-3.

Ansaloni M. (2009). Contrôle politique européen et processus d'européanisation : une comparaison des politiques agro-environnementales anglaises et françaises. Grenoble, Congrès de l'AFSP, 7-9 septembre.

Ansaloni M., Fouilleux E. (2006). Changement de pratiques agricoles : facteurs et modalités d'hybridation technique des exploitations laitières bretonnes. Économie rurale, $\mathrm{n}^{\circ} 292$, mars-avril, p. 6-20.

Arrow K. (1962). The economic implications of learning by doing. American Economic Revue, vol. 29, p. 142-167.

Bartoli P., Boulet D. (1990). Conditions d'une approche en termes de régulation sectorielle. Le cas de la sphère viticole. Cahiers d'économie et de sociologie rurales, $\mathrm{n}^{\circ} 17$, p. 7-38.

Bureau J.-C. (2007). La Politique agricole commune. Collection Repères, La Découverte, $121 \mathrm{p}$.

Clubs-conseils en agro-environnement (2009). Bilan des activités 2007-2008. Évolution des pratiques agro-environnementales des exploitations accompagnées pas les clubs-conseils en agro-environnement (CCAE), $46 \mathrm{p}$.

Darré J.-P. (1996). L'invention des pratiques dans l'agriculture. Vulgarisation et production locale de connaissances. Paris, Karthala, 184 p.

Del'Homme B., Filippi M., Ugaglia A. (2008). Innovations environnementales et proximités : de l'importance des acteurs du territoire pour l'adoption des innovations environnementales en viticulture. $\mathrm{XLV}^{\mathrm{e}}$ Colloque ASRDLF « Territoire et action publique territoriale : nouvelles ressources pour le développement régional », Rimouski, 25-27 août.

Dosi G. (1988). Sources, procedures and micro economic effects of innovation. Journal of economic literature, XXVI, p. 1120-1171.

Dosi G., Teece D., Winter S. (éd.) (1990). Les frontières des entreprises : vers une théorie de la cohérence de la grande entreprise. Revue d'Économie Industrielle, vol. 51, p. 238-254.

Dupeuble T. (2005). Normalisation, ressources cognitives intermédiaires et changement institutionnel : le cas des politiques agro-environnementales en France. INRA SAE2, $7^{\text {es }}$ Journées des Jeunes Chercheurs, 14 et 15 mars.

Dupraz P., Pech M. (2007). Les effets des mesures agri-environnementales. INRA journée du département SAE2, «Évaluation de la Politique agricole commune », 14 juin.

Dron D., Dupraz P., Meynard J.-M. (2003). Grandes cultures. Les dossiers de l'environnement de l'INRA, ${ }^{\circ} 23$, p. 69-92.

Filippi M., Triboulet P. (2006). Typologie des comportements à innover des coopératives, Économie rurale, n ${ }^{\circ} 296$, p. 20-37.

Gasmi N., Grolleau G. (2003). Spécificités des innovations environnementales. Une application aux systèmes agro-alimen- 
taires. Innovations, 2003/2, n ${ }^{\circ} 18$, p. 73-89.

Jollivet M. (2007). La grande transformation de l'agriculture française sous l'œil du sociologue. Économie rurale, $\mathrm{n}^{\circ} 300$, p. 26-29.

Kline L., Rosenberg N. (1986). An overview of innovation. In Landau R., Rosenberg N. (éds.), “The Positive Sum Strategy", National academic press, p. 275-305.

Labarthe P. (2005). Trajectoire d'innovation des services et inertie institutionnelle : dynamique du conseil dans trois agricultures européennes. Géographie, Économie, Société, 2005/3, vol. 73, p. 289-311.

Lundvall B.-A. (1992). National systems of innovation: Towards a theory of innovation and interactive learning. London, $\mathrm{Ed}$. Pinter, $342 \mathrm{p}$.

Lundvall B.-A. (2005). Interactive learning, social capital and economic performance. Conference: Advancing knowledge and the knowledge economy, 10-11 janvier, Washington.

MacKenzie D., Spinardi G. (1995). Tacit Knowledge, Weapons Design and Uninvention of Nuclear Weapons. American Journal of Sociology, vol. 101, $\mathrm{n}^{\circ} 1$, p. 44-99.

Mollard A., Chatellier V., Cordon J.-M., Dupraz P., Jacquet F. (2003). L'agriculture contre l'environnement ? Diagnostic, solutions et perspectives économiques. Annales des Mines, Avril, p. 37-59.

Nonaka I., Toyama R., Konno N. (2000). SECI, Ba and leadership: a unified model of dynamic knowledge creation. Long
Range Planning, vol. 33, $\mathrm{n}^{\circ}$ 1, p 5-34. Nelson R., Winter S. (1982). An Evolutionary Theory of Economic Change. Harvard University press, $454 \mathrm{p}$.

Penrose E. (1959). The Theory of the Growth of the Firm. Oxford Basic Black Well, $296 \mathrm{p}$.

Polanyi M. (1966). The tacit dimension. Peter Smith Publisher, 119 p.

Porter M. (1999). La concurrence selon Porter. Paris, Édition Village Mondial, $475 \mathrm{p}$.

Richardson G. (1972). The Organisation of Industry. The Economic Journal, vol. 82, $\mathrm{n}^{\circ} 2$, p. 883-896.

Rivaud A. (2008). Nouvelle gouvernance agricole et trajectoires productives dans les exploitations : vers une meilleure prise en compte des enjeux environnementaux. Économies et Sociétés, HS n ${ }^{4}$ 2, p. 14611486.

Rivaud A. (2010). Modalités de coordination autour de la ressource en eau douce : enquête auprès des agriculteurs du bassin de la Charente, document de travail CRIEF-TEIR, $\mathrm{n}^{\circ}$ T2010-01.

Teece D., Pisano G. (1998). The dynamic capabilities of firms: an introduction. In Dosi G., Teece D.-J., Chytry J., “Technology, organization and competitiveness", Oxford University Press, p. 193-214.

Vanloqueren G., Baret P.-V. (2009). How agricultural research systems shape a technological regime that develops genetic engineering but locks agroecological innovations, Research Policy, $\mathrm{n}^{\circ} 38$, p. 971-983. 